\title{
Implantação do Prontuário Eletrônico de Paciente ${ }^{1}$
}

\section{Implementation of Electronic Patient Health Record}

\section{Implementación de registro médico electrónico del paciente}

\author{
Marcelo Campara \\ Professor União Educacional do Vale do Aço \\ marcelocampara@hotmail.com
}

\author{
Reinaldo Araujo Alkimin \\ Universidade FUMEC \\ reinaldoalkimim@hotmail.com
}

José Marcos Carvalho de Mesquita

Universidade FUMEC

jose.mesquita@fumec.br

\author{
Cristiana Fernandes De Muylder \\ Universidade Fumec \\ cristiana.muylder@fumec.br
}

\author{
Alexandre Teixeira Dias \\ FACE/FUMEC \\ alexandretdias@fumec.br \\ Jefferson La Falce \\ Centro Universitário UNA \\ jefferson.la.falce@gmail.com
}

\section{Resumo}

Em cenário onde a tecnologia influência no desempenho do funcionário esta pesquisa visa descobrir quais são as expectativas dos profissionais da saúde em relação ao uso do Prontuário Eletrônico do Paciente (PEP). Para isso, foram realizadas 19 entrevistas com médicos, enfermeiros e técnicos de enfermagem, pois serão eles os usuários do sistema. O questionário foi baseado em modelo de paradoxos que é utilizado para mensurar como o uso da tecnologia pode gerar efeitos positivos ou negativos àqueles que adotam. Tais paradoxos dividem-se em perguntas que buscam afirmar uma questão ou desmenti-la, confirmando ou desconstruindo o paradoxo testado. Percebeu-se através da pesquisa que a equipe da área da saúde está com uma expectativa positiva de que o PEP poderá trazer controle, liberdade, competência, eficiência, satisfação, integração e engajamento. Alguns respondentes tiveram percepção negativa acerca da utilização do PEP. Ressalta-se que a pesquisa contribuiu para a discussão da ferramenta bem como instiga novos estudos acerca de sua adoção e sugere comparação de resultados previstos e realizados em casos de uso do PEP.

Palavras Chaves: Prontuário eletrônico do paciente, Tecnologia da informação e comunicação em Saúde, Registro eletrônico de saúde.

\begin{abstract}
In a scenario where technology influences on employee performance this research aims to find out what the health professionals think about using Patient Electronic Database (PEP). The research was made with 19 doctors and paramedics. The form was based on paradoxes model that measure the positive and negative effects of technology use. Such paradoxes are divided into questions that seek to affirm a question or contradict it, confirming or deconstructing the paradox tested. According the research there is a positive feeling about the PEP's use and the control, freedom, competence, efficiency, satisfaction, integration and engagement results. Some people interviewed had negative perceptions about the PEP's use. The research contributed to the theme discussion and about PEP's expected performance.
\end{abstract}

Key Words: Patient electronic database, Healthy information and communication technology, Healthy electronic database.

\section{Resumen}

\footnotetext{
${ }^{1}$ Submetido em 19 de setembro de 2013. Aceito em 02 de dezembro de 2013. O artigo foi avaliado segundo o processo de duplo anonimato além de ser avaliado pelo editor. Editores responsáveis: Márcio Augusto Gonçalves e Lucas Maia dos Santos. Reprodução parcial ou total e trabalhos derivativos permitidos com a citação apropriada da fonte.
} 
Revista de Administração Hospitalar, v.10, n.3, pp. 61-74, setembro/dezembro, 2013/ Marcelo Campara, Reinaldo Araujo Alkimin, Cristiana Fernandes De Muylder, Alexandre Teixeira Dias, José Marcos Carvalho de Mesquita, Jefferson La Falce

Este estudio investiga cuáles son las expectativas de los profesionales de la salud en relación con la utilización de Historia Clínica Electrónica (HCE) presentes en el escenario actual, donde la influencia de la tecnología en el desempeño del empleado. celebró 19 entrevistas con médicos, enfermeras y técnicos de enfermería, ya que serán los usuarios del sistema. El cuestionario se basa en el modelo de paradojas que se utiliza para medir el uso de la tecnología puede generar efectos positivos o negativos a los que adoptan. Estas paradojas se dividen en cuestiones que tratan de afirmar una pregunta o contradecirla, confirmar o deconstruir esta paradoja. Era capaz de darse cuenta de los resultados de la investigación que el personal de salud poseen una expectativa positiva de que la PEP puede llevar el control, la libertad, la competencia, la eficiencia, la satisfacción, la integración y la participación. Algunos de los encuestados tuvieron percepciones negativas sobre el uso de PEP. Cabe destacar que la investigación contribuyó a la discusión de la herramienta y alienta nuevos estudios sobre su adopción y sugiere comparar esperado y ha actuado en los casos de uso de PEP.

Palabras clave: Historia clínica electrónica, Tecnologías de la Información y Comunicación en Salud, Historia Clínica Electrónica.

\section{Introdução}

Atualmente, a informatização não é mais vista apenas como auxílio à execução de tarefas, mas como ponto chave que pode gerar diferencial competitivo e fazer diferença entre sucesso e fracasso na administração e interpretação das informações. Mesmo assim, são percebidos problemas na adoção, implantação e aceitação de sistemas informatizados. Relatos de empresas podem ser encontrados no cotidiano e disponíveis via Internet.

Um dos fatores que podem causar grande desconforto e levar um projeto de implantação de software em níveis desfavoráveis é a insatisfação do usuário para com o sistema, afinal ele geralmente é o mais afetado. Os usuários, na maioria das vezes, buscam entender a real necessidade do sistema e projetam seu próprio futuro depois da implantação de sistemas de informação. Várias preocupações estão presentes nesse momento, como possível diminuição de funcionários, incapacidade de operar o sistema, qualidade do sistema, dentre outros.

Segundo a Sociedade Brasileira de Informática em Saúde (SBIS), a utilização da Tecnologia da Informação e Comunicação em Saúde (TICS) cresce a cada dia. Hoje são inúmeras as possibilidades, os recursos e os benefícios que a informática pode trazer para a área de saúde, especialmente para o médico.

O Prontuário Eletrônico do Paciente (PEP) é a principal ferramenta de Tecnologia da Informação e Comunicação em Saúde (TICS) que o médico precisa ou precisará lidar nas suas atividades diárias, seja no consultório, centro diagnóstico ou hospital.

No entanto, é fundamental que o médico utilize uma ferramenta que assegure o registro da história clínica e exame físico, bem como na solicitação de exames e prescrição dentro de preceitos éticos e legais.

Outro conceito importante, neste cenário de informatização da área de saúde, é o Registro Eletrônico de Saúde (RES) que permite o armazenamento e o compartilhamento seguro das informações de um paciente.

Os sistemas devem adotar mecanismos de segurança capazes de garantir autenticidade, confidencialidade e integridade das informações de saúde. A certificação digital é a tecnologia que melhor provê estes mecanismos.

Com o intuito de estabelecer as normas, padrões e regulamentos para o PEP/RES no Brasil, o Conselho Federal de Medicina (CFM) e a Sociedade Brasileira de Informática em Saúde (SBIS) estabeleceram convênio de cooperação técnico-científica que está em vigência desde 2002.

Esse convênio propiciou a criação de um processo de certificação de sistemas de Registro Eletrônico de Saúde, com o estabelecimento dos requisitos obrigatórios e, acompanhando a legislação federal para documento eletrônico, reforçou a obrigatoriedade no uso de certificação digital (assinatura eletrônica) para a validade ética e jurídica de um 
Revista de Administração Hospitalar, v.10, n.3, pp. 61-74, setembro/dezembro, 2013/ Marcelo Campara, Reinaldo Araujo Alkimin, Cristiana Fernandes De Muylder, Alexandre Teixeira Dias, José Marcos Carvalho de Mesquita, Jefferson La Falce

PEP/RES. Outro marco regulatório relevante neste cenário, foi a publicação da Resolução CFM No $1821 / 2007$.

E fevereiro de 2012, a Sociedade Brasileira de Informática em Saúde (SBIS, 2012) publicou em seu site um documento denominado "Cartilha sobre Prontuário Eletrônico - a Certificação de Sistemas de Registro Eletrônico de Saúde". Este documento, de acordo com a SBIS (2012) tem o intuito de auxiliar os médicos a compreender melhor os conceitos-chaves da certificação de software e certificação digital com exemplificação na área de registros médicos. Por meio da cartilha, o profissional médico e os demais interessados podem compreender significados e regras para um prontuário digital, através uma linguagem facilitada. Diante do uso destas tecnologias da informação no contexto da área de saúde temse o problema que motivou este artigo: qual a percepção dos profissionais da área de saúde no uso de tecnologia Prontuário Eletrônico do Paciente/Registro Eletrônico de Saúde?

O objetivo geral foi entender quais são as expectativas dos profissionais da saúde médicos, enfermeiros e técnicos em enfermagem - com relação à implantação do prontuário eletrônico. Especificamente, pretendeu-se identificar o grau de variação da expectativa em relação a função e o tempo de experiência dos profissionais da saúde.

Para isto contou-se com pesquisa descritiva baseada em modelo de Mick \& Fournier (1998) onde foram entrevistados 19 profissionais de saúde em duas semanas de pesquisa.

O presente artigo divide-se além desta introdução do desenvolvimento teórico, metodologia, resultados e considerações finais, seguidas das referências usadas.

\section{Prontuário Eletrônico}

O prontuário médico é um documento desenvolvido por médicos e enfermeiros e usados por eles para registrar os eventos clínicos prestados ao pacientes (MASSAD, MARIN e AZEVEDO NETO, 2003).

De acordo com Pinto (2006), os registros das informações de pacientes e doentes ocorrem desde a idade antiga. Ainda de acordo com o autor, os primeiros registros foram atribuídos aos egópicios, especificamente ao médico Imhotep (PINTO, 2006).

A revisão da literatura mostra que as informações dos pacientes eram feitos em um documento único por ordem cronológica por médico e atendimento. Desta forma, era difícil para os profissionais de saúde resgatar informações do paciente quando necessário. Em um momento posterior os prontuários foram individualizados e cada paciente passou a ter o seu.

Na visão de Massad, Marin e Azevedo Neto (2003, p. 6) o prontuário eletrônico do paciente - PEP é "um meio físico, um repositório onde todas as informações de saúde, clínicas e administrativas, ao longo da vida de um indivíduo estão armazenadas". O repositório, citado pelo autor, é o meio eletrônico de armazenamento, que pode ser uma base de dados acessada por uma interface de aplicação. Porém, a migração dos prontuários em suporte tradicional para o eletrônico introduziu várias mudanças no contexto da saúde, tanto para os profissionais, como para os clientes e também para os gestores de serviços de informação das organizações de saúde. No âmbito deste artigo iremos analisar as mudanças para os profissionais de saúde (MASSAD, MARIN e AZEVEDO NETO, 2003).

O PEP é composto por uma estrutura física e lógica. A estrutura física equivale a categoria de tópicos relativas ao paciente, convênio e internação, além do controle de saída da organização de saúde. A estrutura lógica por sua vez, trata das informações relacionadas à identificação do paciente, cuidados de saúde e prescrição.

De acordo com Pinto (2006) as categorias de informação do PEP são descritiva, operacionais e de advertência (QUADRO 1).

O uso de PEP pode gerar benefícios que são apontados por alguns autores. Dentre estes, Van Bemmel (1997) indica que o prontuário eletrônico é melhor, pois se pode permitir 
Revista de Administração Hospitalar, v.10, n.3, pp. 61-74, setembro/dezembro, 2013/ Marcelo Campara, Reinaldo Araujo Alkimin, Cristiana Fernandes De Muylder, Alexandre Teixeira Dias, José Marcos Carvalho de Mesquita, Jefferson La Falce

acesso ao mesmo por diferentes meios e em locais diferentes. De acordo com Sitting (1994), as vantagens de usar prontuário eletrônico são acesso remoto e simultâneo legibilidade (as letras difíceis de compreensão não são usadas, pois os textos são digitados), ocorre segurança dos dados por meio de técnicas de backup, preocupa-se com confidencialidade a partir do momento que o acesso é monitorado e restrito e permite incluir informações de exames de imagens e resultados clínicos importantes para o diagnóstico adequado.

Quadro 1 - Diferentes categorias de informação do PEP categorais de informações

\begin{tabular}{|l|l|}
\hline Categorias & Definições \\
\hline Descritivas & $\begin{array}{l}\text { Informações que descrevem o estado atual da realidade. "Paciente fez uso de 01 comp. } \\
\text { Prometazina 25 mg.", "Cliente cooperativo, respondendo as solicitaçôes". }\end{array}$ \\
\hline Operacionais & $\begin{array}{l}\text { Informações cujo objetivo é oferecer as condições para gerir as situações dadas, ou } \\
\text { seja, agir e/ou reagir sobre elas. "Tomar 01 cp de } 12 \text { /12 h.", }\end{array}$ \\
\hline De advertência & $\begin{array}{l}\text { Informações cujo objetivo é chamar atenção para um fato. "Paciente apresenta perda } \\
\text { de peso", "Paciente apresentando lesões pruriginosas pelo corpo" }\end{array}$ \\
\hline
\end{tabular}

Fonte: Pinto, 2006, p. 11

De acordo com McDonald e Barnett (1990) ocorrem algumas desvantagens no uso do prontuário eletrônico: investimento alto em tecnologia; treinamento de funcionários e equipe médica envolvida e dependência do uso de sistemas e infraestrutura de computação.

Existe diferença entre o PEP e o Registro Eletrônico de Saúde (RES). Segundo Santos (2011), o RES consolida informações de paciente de diversos sistemas PEPs em uma organizaçao de âmbito global, enquanto o PEP está restrito a uma única organização de saúde.

\section{Registro Eletrônico de Saúde}

Desde 2007, o Governo Brasileiro desenvolve ações e discute estratégias de desenvolvimento do setor de saúde e dentro desta pauta ocorrem discussões acerca de melhorias de qualidade do gasto público e gestão de recursos na área da saúde (BRASIL, 2013).

O número crescente de ferramentas informatizadas para a área de saúde bem como novos procedimentos clínicos, cirúrgicos, laboratoriais, etc agrava a complexidade informacional fazendo que o histórico dos eventos ocorridos com o paciente seja relevante.

Os dados organizados e registrados em banco de dados com todas as informações da vida de um paciente podem compor o que é denominado Registro Eletrônico de Saúde (RES). Este depósito de informações exige critérios de segurança, qualidade e velocidade de acesso (BUI et al., 2001). Existem autoridades que controlam e monitoram a qualidade destes registros que seguem normas como a ISO/TR 20514 de 2005.

Segundo Santos (2011, p. 13), o "RES pode conter dados originados de várias instituições de saúde, de maneira a possibilitar um histórico integrado de atendimentos dos pacientes. A disponibilidade da informação de saúde integrada, no local onde a assistência médica é realizada, pode modificar o cenário de saúde seja em uma região, país ou, até mesmo, de forma globalizada.".

O autor ainda ressalta que “... o RES, de um componente central na construção de um sistema integrado de saúde. Como visto, constitui um repositório para pesquisa e melhoria nas condições de tratamento de pacientes. Mas pode também ser útil para a definição de políticas de saúde pública." (SANTOS, 2011, p.20).

\section{Metodologia}


Revista de Administração Hospitalar, v.10, n.3, pp. 61-74, setembro/dezembro, 2013/ Marcelo Campara, Reinaldo Araujo Alkimin, Cristiana Fernandes De Muylder, Alexandre Teixeira Dias, José Marcos Carvalho de Mesquita, Jefferson La Falce

Optou-se pela utilização do método de estudo de caso simples, no qual o indivíduo é a unidade de análise, pela aplicação de questionário estruturado baseado nos paradoxos tecnológicos de Mick e Fournier (1998).

O conceito de paradoxo originou-se com os filósofos da antiguidade e, desde então, muitos pesquisadores têm estudado este conceito (DE VRIES, 1995; SABELIS 1996; HATCH e EHRLICH, 1993; MURNIGHAN e CONLON, 1991; VINCE e BROUSSINE, 1996; EISENHARDT e WESCOTT, 1988). Todo paradoxo é uma afirmação e uma negação de forma fundamentada e válida, na qual a verdade de uma implica necessariamente na falsidade da outra e vice-versa.

Cada um dos oito paradoxos, propostos por Mick e Fournier (1998), foram tratados em duas ou mais perguntas, que representassem o antagonismo associado àquele paradoxo no questionário, que fez uso da escala de Likert, na intenção de avaliar a concordância dos entrevistados em cada questão (QUADRO 2).

\begin{tabular}{|c|c|}
\hline P1:Controle/Caos & $\begin{array}{l}\text { A tecnologia tanto pode facilitar a ordem e o controle das } \\
\text { tarefas e situaçōes, quanto pode provocar desordem, } \\
\text { descontrole e revolta. }\end{array}$ \\
\hline P2:Liberdade/Escravidão & $\begin{array}{l}\text { A tecnologia tanto pode facilitar a independência e reduzir } \\
\text { restrições, quanto pode provocar dependência e mais } \\
\text { restriçōes. }\end{array}$ \\
\hline P3:Novo/Obsoleto & $\begin{array}{l}\text { A tecnologia tanto pode trazer novos beneficios decorrentes } \\
\text { do avanço do conhecimento, quanto pode estar ultrapassada } \\
\text { no momento em que se torna acessivel ao consumidor. }\end{array}$ \\
\hline P4:Competência/Incompetência & $\begin{array}{l}\text { A tecnologia tanto pode trazer sentido de eficiência e } \\
\text { inteligência, quanto pode provocar sentimentos de } \\
\text { incompetência e ignorância, em decorrência da complexidade } \\
\text { e dificuldade de uso. }\end{array}$ \\
\hline P5:Eficiência/Ineficiência & $\begin{array}{l}\text { A tecnologia tanto possibilita mais rapidez e menos esforço } \\
\text { para a realização de certas tarefas, quanto pode requerer mais } \\
\text { tempo e mais esforço, em outras. }\end{array}$ \\
\hline P6:Satisfação/Criação de Necessidades & $\begin{array}{l}\text { A tecnologia tanto pode facilitar a satisfação de desejos e } \\
\text { necessidades, quanto pode tornar conscientes desejos e } \\
\text { necessidades ainda não reconhecidos. }\end{array}$ \\
\hline P7:Integração/ssolamento & $\begin{array}{l}\text { A tecnologia tanto pode facilitar a interação entre pessoas, } \\
\text { quanto pode provocar a separação delas. }\end{array}$ \\
\hline P8:Engajamento/Desengajamento & $\begin{array}{l}\text { A tecnologia tanto pode facilitar o envolvimento, o fluxo e a } \\
\text { ativação das pessoas, quanto pode provocar acomodação, } \\
\text { passividade e falta de conexão. }\end{array}$ \\
\hline
\end{tabular}

Quadro 2 - Detalhamento dos paradoxos

Fonte: Mick e Fournier (1998)

O questionário usado contem 19 perguntas, que foram distribuídas de forma aleatória, na tentativa de minimizar a percepção do paradoxo pelo entrevistado.

Visando facilitar os procedimentos de análise das informações coletadas nos questionários, foi criada uma base de dados onde todas as informações foram inseridas. Esse 
Revista de Administração Hospitalar, v.10, n.3, pp. 61-74, setembro/dezembro, 2013/ Marcelo Campara, Reinaldo Araujo Alkimin, Cristiana Fernandes De Muylder, Alexandre Teixeira Dias, José Marcos Carvalho de Mesquita, Jefferson La Falce

procedimento propiciou várias consultas com maior facilidade e o desenvolvimento de gráficos que auxiliaram na interpretação dos resultados.

O público-alvo foi divido em níveis estratégico, gerencial e operacional, sendo composto por sete médicos, seis enfermeiros e seis técnicos de enfermagem respectivamente. Este público corresponde a um grupo de profissionais de saúde de dois hospitais do estado de Minas Gerais e o critério de escolha destes foi acessibilidade.

\section{Resultados}

$\mathrm{Na}$ primeira fase de análise, pode-se testar a aderência das respostas em relação aos paradoxos propostos, o quadro dois define a relação entre as perguntas e os paradoxos.

\begin{tabular}{|c|c|c|}
\hline Paradoxo & Conceito & Pergunta \\
\hline P1: Controle / Caos & $\begin{array}{l}\text { A tecnologia tanto pode facilitar a } \\
\text { ordem e o controle das tarefas, } \\
\text { quanto pode provocar desordem, } \\
\text { descontrole e revolta. }\end{array}$ & $\begin{array}{l}\text { 1) Em sua opinião a utilização do Prontuário } \\
\text { Eletrônico poderia aumentar o controle dos } \\
\text { dados? } \\
\text { 9) Em sua opinião programa pode facilitar as } \\
\text { tarefas rotineiras? } \\
\text { 16) Em sua opinião o Prontuário Eletrônico é } \\
\text { desnecessário para o controle dos dados dos } \\
\text { pacientes? }\end{array}$ \\
\hline P2: Liberdade / Escravidão & $\begin{array}{l}\text { A tecnologia tanto pode facilitar a } \\
\text { independência e reduzir } \\
\text { restrições, quanto pode provocar } \\
\text { dependência e mais restrições. }\end{array}$ & $\begin{array}{l}\text { 6) Em sua opinião após a implantação do } \\
\text { sistema o trabalho dos profissionais poderão } \\
\text { ficar dependente do Prontuário Eletrônico? } \\
\text { 10) Em sua opinião este sistema pode facilitar } \\
\text { o trabalho dos profissionais tornando as } \\
\text { atividades mais independentes? } \\
\text { 17) Em sua opinião o programa pode trazer } \\
\text { mais burocratização nos processos, } \\
\text { dificultando a rotina de trabalho? }\end{array}$ \\
\hline P3: Novo/obsoleto & $\begin{array}{l}\text { A tecnologia tanto pode trazer } \\
\text { novos benefícios decorrentes do } \\
\text { avanço do conhecimento, } \\
\text { quanto pode estar ultrapassada } \\
\text { no momento em que se torna } \\
\text { acessível ao consumidor. }\end{array}$ & $\begin{array}{l}\text { 7) Em países desenvolvidos o Prontuário } \\
\text { Eletrônico já é uma realidade. Em sua opinião } \\
\text { o sistema já deveria ter sido implementado no } \\
\text { Brasil? } \\
\text { 11) Em sua opinião após a implantação do } \\
\text { sistema, novos conhecimentos poderão ser } \\
\text { desenvolvidos? }\end{array}$ \\
\hline $\begin{array}{l}\text { P4: Competência / } \\
\text { Incompetência }\end{array}$ & $\begin{array}{l}\text { A tecnologia tanto pode trazer } \\
\text { sentido de eficiência e } \\
\text { inteligência, quanto pode } \\
\text { provocar sentimentos de } \\
\text { incompetência e ignorância, em } \\
\text { decorrência da complexidade e } \\
\text { dificuldade de uso. }\end{array}$ & $\begin{array}{l}\text { 8) Em sua opinião se os profissionais da área } \\
\text { não estiverem preparados para operar o } \\
\text { programa poderá gerar sentimento de } \\
\text { incompetência? } \\
\text { 18) Em sua opinião as entidades competentes } \\
\text { precisarão fazer um programa de capacitação } \\
\text { para que os profissionais envolvidos tenham } \\
\text { domínio completo do Sistema? }\end{array}$ \\
\hline P5: Eficiência / Ineficiência & $\begin{array}{l}\text { A tecnologia tanto possibilita } \\
\text { mais rapidez e menos esforço } \\
\text { para a realização de certas tarefas, } \\
\text { quanto pode requerer mais tempo } \\
\text { e mais esforço, em outras. }\end{array}$ & $\begin{array}{l}\text { 12) Em sua opinião a implantação do programa } \\
\text { trará mais rapidez na execução das tarefas? } \\
\text { 2) Em sua opinião o programa gerará menos } \\
\text { esforço por parte dos profissionais na execução } \\
\text { das tarefas? }\end{array}$ \\
\hline $\begin{array}{l}\text { P6: Satisfação / Criação de } \\
\text { necessidades }\end{array}$ & $\begin{array}{l}\text { A tecnologia tanto pode facilitar a } \\
\text { satisfação de desejos e } \\
\text { necessidades, quanto pode tornar } \\
\text { conscientes desejos e } \\
\text { necessidades ainda não } \\
\text { reconhecidos. }\end{array}$ & $\begin{array}{l}\text { 13) Em sua opinião o programa pode agregar } \\
\text { valor ao trabalho dos profissionais? } \\
\text { 3) Em sua opinião o programa pode auxiliar os } \\
\text { pacientes em sua saúde pessoal? } \\
\text { 19) Em sua opinião o sistema pode gerar } \\
\text { futuras necessidades para os envolvidos? }\end{array}$ \\
\hline P7: Integração / Isolamento & $\begin{array}{l}\text { A tecnologia tanto pode facilitar a } \\
\text { interação entre pessoas, quanto } \\
\text { pode provocar a separação delas. }\end{array}$ & $\begin{array}{l}\text { 14) Em sua opinião a implantação do sistema } \\
\text { poderá gerar mais integração entre os } \\
\text { profissionais? } \\
\text { 4) Em sua opinião a implantação do sistema }\end{array}$ \\
\hline
\end{tabular}


Revista de Administração Hospitalar, v.10, n.3, pp. 61-74, setembro/dezembro, 2013/ Marcelo Campara, Reinaldo Araujo Alkimin, Cristiana Fernandes De Muylder, Alexandre Teixeira Dias, José Marcos Carvalho de Mesquita, Jefferson La Falce

\begin{tabular}{|l|l|l|}
\hline & & $\begin{array}{l}\text { pode estimular o isolamento entre medico e } \\
\text { paciente? }\end{array}$ \\
\hline $\begin{array}{l}\text { P8: Engajamento / } \\
\text { Desengajamento }\end{array}$ & $\begin{array}{l}\text { A tecnologia tanto pode facilitar o } \\
\text { envolvimento, o fluxo e a } \\
\text { ativação das pessoas, quanto pode } \\
\text { provocar acomodaçãão após a implantação do } \\
\text { passividade e falta de conexão. }\end{array}$ & $\begin{array}{l}\text { Prontuário Eletrônico os profissionais se } \\
\text { engajarão em prol do sistema? } \\
\text { 5) Em sua opinião sistema pode causar } \\
\text { acomodação dos profissionais? }\end{array}$ \\
\hline
\end{tabular}

\section{Quadro 2: Relação perguntas / paradoxo}

Fonte: Dados da pesquisa

O Quadro 3 possibilita uma visão do panorama geral do resultado da pesquisa, com um total de 19 entrevistados divididos em seis Enfermeiros, sete Médicos e seis Técnicos em Enfermagem.

\begin{tabular}{|c|c|c|c|c|c|}
\hline Pergunta & Resposta & Quantidade & Pergunta & Resposta & Quantidade \\
\hline 1 & A & 1 & 10 & $\mathrm{C}$ & 2 \\
\hline 1 & $\bar{D}$ & 5 & 10 & $\mathrm{D}$ & 6 \\
\hline 1 & $\mathrm{E}$ & 12 & 10 & $E$ & 7 \\
\hline 2 & $\mathrm{~A}$ & 2 & 11 & $\mathrm{C}$ & 1 \\
\hline 2 & $\mathrm{D}$ & 11 & 11 & $\mathrm{D}$ & 9 \\
\hline 2 & $E$ & 5 & 11 & $E$ & 8 \\
\hline 3 & $\mathrm{~A}$ & 1 & 12 & $\mathrm{~A}$ & 2 \\
\hline 3 & B & 2 & 12 & B & 1 \\
\hline 3 & $\mathrm{C}$ & 5 & 12 & $\mathrm{D}$ & 8 \\
\hline 3 & D & 6 & 12 & $\mathrm{E}$ & 7 \\
\hline 3 & $\mathrm{E}$ & 4 & 13 & $\mathrm{~A}$ & 3 \\
\hline 4 & $\mathrm{~A}$ & 9 & 13 & B & 2 \\
\hline 4 & $\mathrm{~B}$ & 6 & 13 & $\mathrm{C}$ & 2 \\
\hline 4 & $\mathrm{C}$ & 2 & 13 & $\mathrm{D}$ & 4 \\
\hline 4 & $\mathrm{D}$ & 1 & 13 & $\mathrm{E}$ & 7 \\
\hline 5 & $\mathrm{~A}$ & 5 & 14 & $\mathrm{~A}$ & 2 \\
\hline 5 & B & 6 & 14 & B & 3 \\
\hline 5 & $\mathrm{C}$ & 1 & 14 & $\mathrm{C}$ & 1 \\
\hline 5 & $\mathrm{D}$ & 6 & 14 & $\mathrm{D}$ & 9 \\
\hline 6 & $\bar{A}$ & 3 & 14 & $\mathrm{E}$ & 3 \\
\hline 6 & $\mathrm{~B}$ & 6 & 15 & $\mathrm{~A}$ & 3 \\
\hline 6 & $\mathrm{C}$ & 1 & 15 & B & $\overline{1}$ \\
\hline 6 & $\mathrm{D}$ & 5 & 15 & $\mathrm{D}$ & 12 \\
\hline 6 & $\mathrm{E}$ & 2 & 15 & $E$ & 2 \\
\hline 7 & $\mathrm{~A}$ & 3 & 16 & $\mathrm{~A}$ & 12 \\
\hline 7 & D & 3 & 16 & B & 2 \\
\hline 7 & $E$ & 12 & 16 & $\mathrm{D}$ & 2 \\
\hline 8 & A & 3 & 16 & $\mathrm{E}$ & 2 \\
\hline 8 & $\mathrm{~B}$ & 1 & 17 & $\mathrm{~A}$ & 6 \\
\hline 8 & $\mathrm{C}$ & 3 & 17 & B & 6 \\
\hline 8 & $\mathrm{D}$ & 9 & 17 & $\mathrm{D}$ & 5 \\
\hline 8 & E & 2 & 17 & E & 1 \\
\hline 9 & A & 1 & 18 & A & 2 \\
\hline 9 & B & 3 & 18 & D & 5 \\
\hline 9 & $\mathrm{D}$ & 6 & 18 & $\mathrm{E}$ & 11 \\
\hline 9 & $\mathrm{E}$ & 8 & 19 & $\mathrm{~B}$ & 1 \\
\hline 10 & $\mathrm{~A}$ & 1 & 19 & $\mathrm{C}$ & 1 \\
\hline \multirow[t]{2}{*}{10} & B & 2 & 19 & D & 7 \\
\hline & & & 19 & $E$ & 9 \\
\hline
\end{tabular}

Quadro 3: Panorama geral das respostas

Fonte: Dados da pesquisa 
Revista de Administração Hospitalar, v.10, n.3, pp. 61-74, setembro/dezembro, 2013/ Marcelo Campara, Reinaldo Araujo Alkimin, Cristiana Fernandes De Muylder, Alexandre Teixeira Dias, José Marcos Carvalho de Mesquita, Jefferson La Falce

De acordo com GRAF. 1, pode-se perceber a preferência dos usuários quanto as questões levantadas.

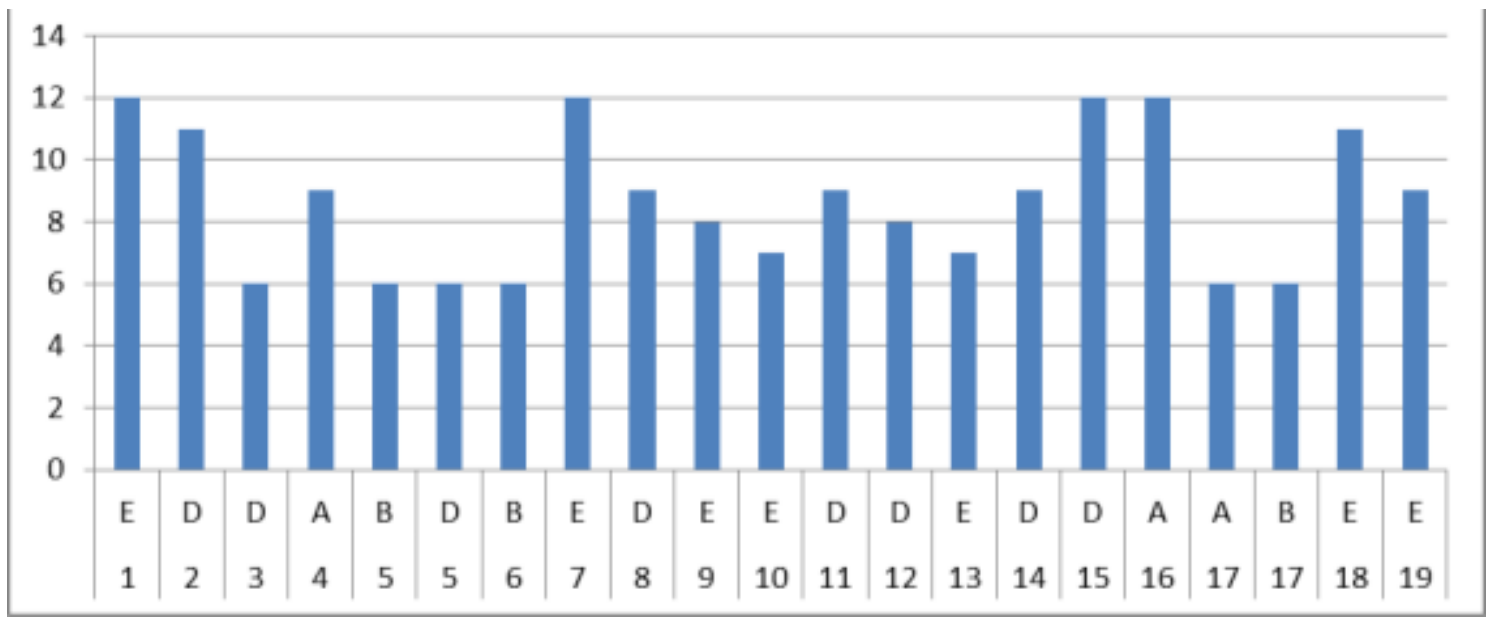

Gráfico 1 - Preferência das questões por parte dos usuários.

Fonte: Dados da pesquisa

Constata-se que existe uma concordância de $63 \%$ em relação às perguntas 1, 7, 15 e 16. Estas perguntas representam, respectivamente, os paradoxos controle/caos, novo/obsoleto, engajamento/desengajamento e controle/caos. Observa-se que as questões 1 e 16 pertencem ao mesmo paradoxo. Na pergunta 1, a maioria dos respondentes concordam totalmente que o PEP poderia aumentar o controle dos dados, enquanto que na pergunta 16 a maioria dos respondentes não concordam totalmente que o PEP é desnecessário para o controle dos dados.

Portanto, neste sentido o paradoxo foi satisfeito.

Outra visão relevante é analisar as preferências de cada função em relação às perguntas, assim o GRAF. 2, GRAF. 3 e GRAF. 4 auxiliam nesta percepção selecionando qual foi a maior quantidade de repostas por pergunta de Médicos, Enfermeiros e Técnicos em Enfermagem.

Considerando que as respostas " $\mathrm{D}$ " $\mathrm{e}$ " $\mathrm{E}$ " tendem a confirmar o lado positivo do paradoxo e as respostas " $\mathrm{A}$ " e "B" tendem para a parte negativa do paradoxo, então tem-se doze respostas parecidas que representam $63 \%$ do total de perguntas.

Percebe-se então que a função tem pouca influência na percepção que os usuários possuem da adoção do PEP. 
Revista de Administração Hospitalar, v.10, n.3, pp. 61-74, setembro/dezembro, 2013/ Marcelo Campara, Reinaldo Araujo Alkimin, Cristiana Fernandes De Muylder, Alexandre Teixeira Dias, José Marcos Carvalho de Mesquita, Jefferson La Falce

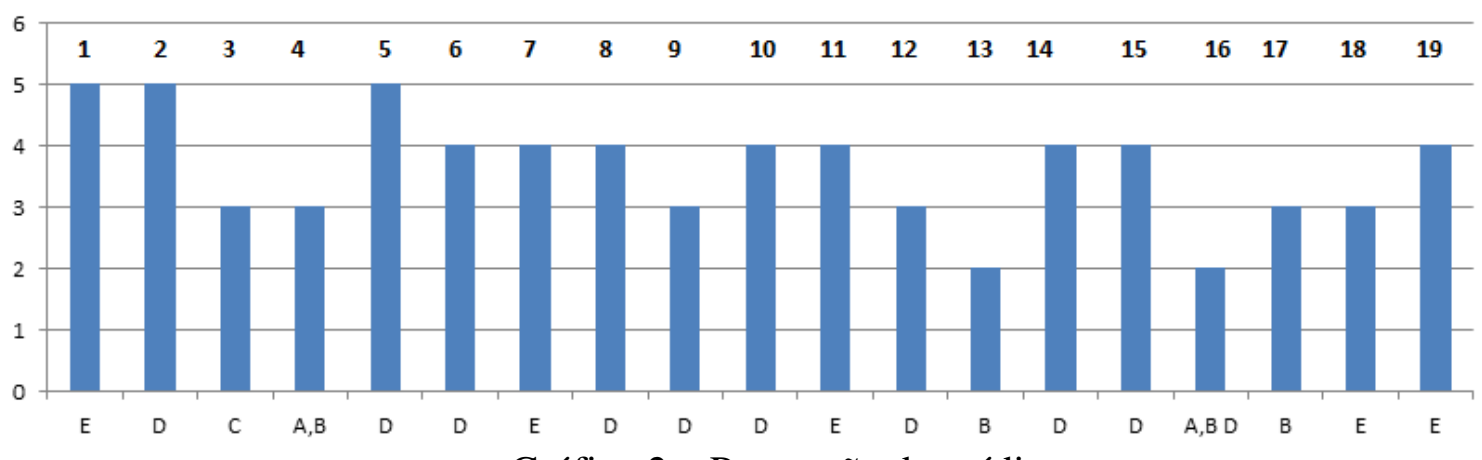

Gráfico 2 - Percepção de médicos

Fonte: Dados da pesquisa
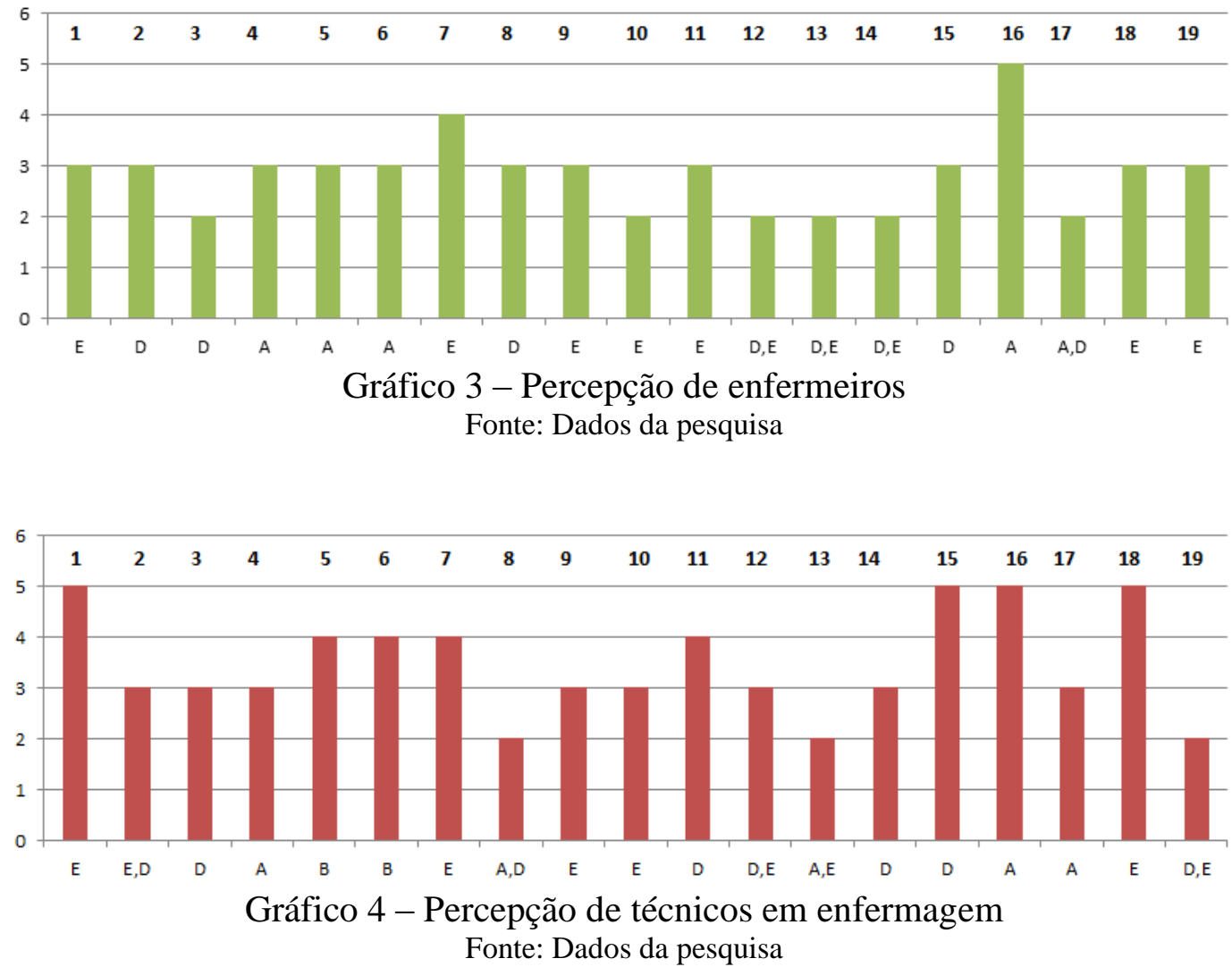

O GRAF. 5 demonstra uma análise em relação ao tempo de experiência do profissional que respondeu a pesquisa.

Percebe-se que as respostas " $D$ " e "E" tendem a confirmar o lado positivo do paradoxo e as respostas "A" $\mathrm{e}$ "B" tendem para a parte negativa do paradoxo.

Pode-se denominar de "parte positiva", pois se relaciona com Controle, Liberdade, Novo, Competência, Eficiência, Satisfação, Integração e Engajamento. Em contrapartida, denomina-se por "parte negativa", pois se relaciona com Caos, Escravidão, Obsoleto, Incompetência, Ineficiência, Criação de necessidades, Isolamento e Desengajamento. 
Revista de Administração Hospitalar, v.10, n.3, pp. 61-74, setembro/dezembro, 2013/ Marcelo Campara, Reinaldo Araujo Alkimin, Cristiana Fernandes De Muylder, Alexandre Teixeira Dias, José Marcos Carvalho de Mesquita, Jefferson La Falce

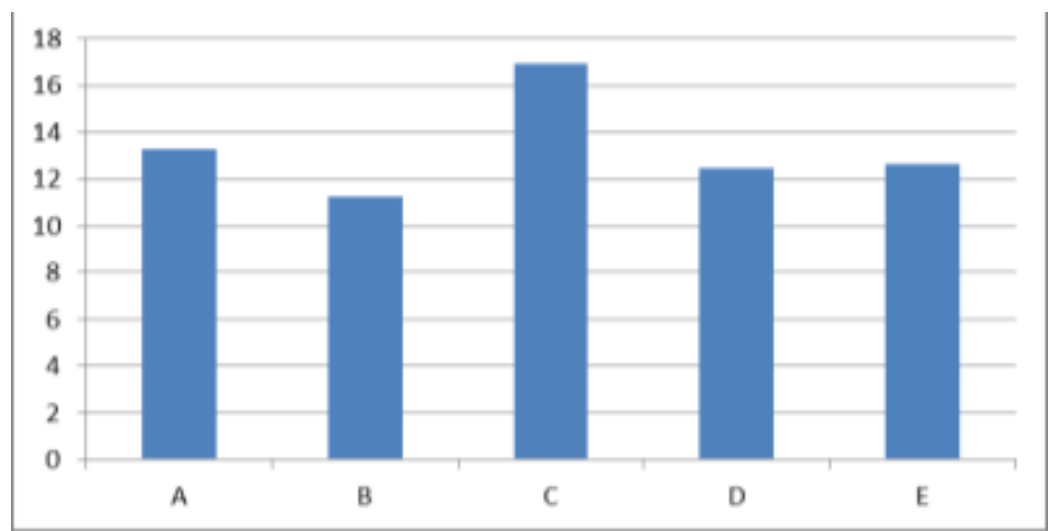

Gráfico 5 - Média do tempo de experiência do profissional Fonte: Dados da pesquisa

Estas relações podem estar relacionadas à idade do profissional e seu perfil quanto a aceitação no uso de tecnologia. Quando se calcula a média da experiência do profissional e as respostas dadas, percebe-se não haver discrepância da média em relação às respostas, sendo mínima a variação encontrada.

Desta forma, desconstrói-se, nesta amostra de estudo de caso, tal pré-conceito em relação à idade do profissional e a propensão a aceitação de novos recursos tecnológicos.

Em outra fase da análise dos dados, se verificou as respostas dos entrevistados em relação aos paradoxos propostos.

O GRAF. 6 apresenta o número de respostas de cada pergunta, assim se vincula essas respostas aos paradoxos e pode-se identificar a qual parte do paradoxo o grupo de entrevistados se refere.

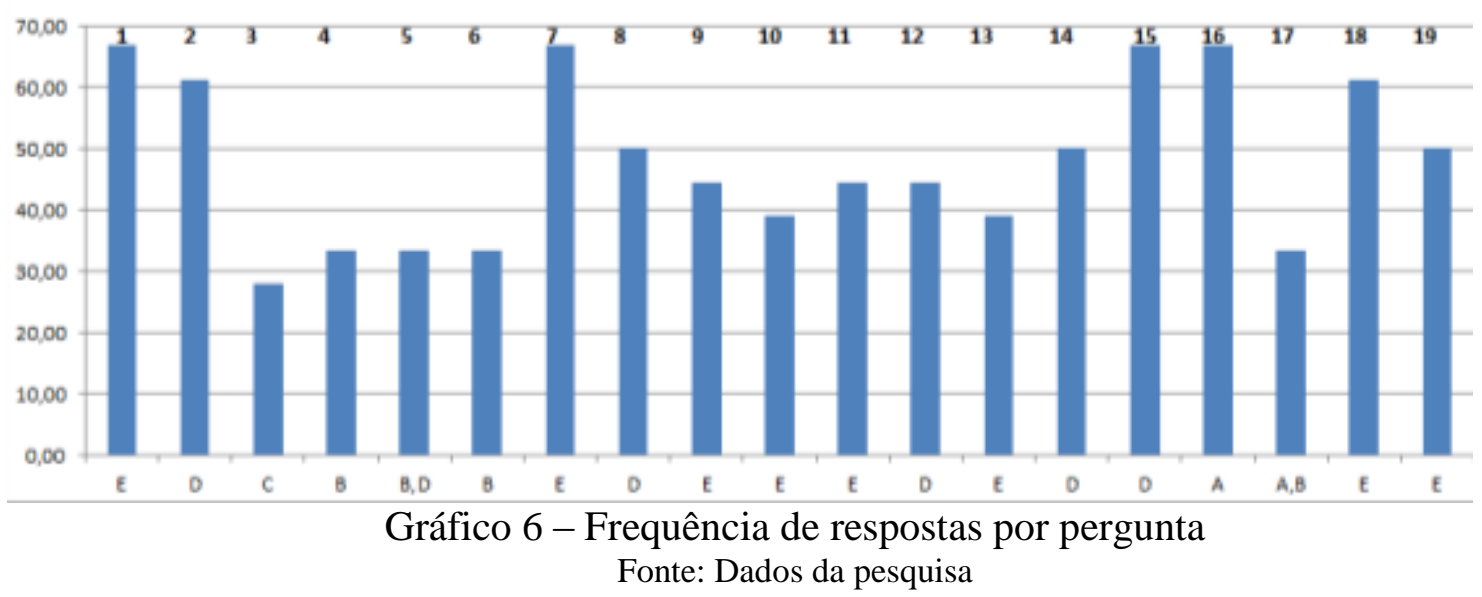

\section{Discussões e Paradoxos}

Nesta fase foi feita uma comparação dos resultados por paradoxo proposto pelo modelo adotado na pesquisa.

Paradoxo um: controle/caos

As perguntas 1, 9 e 16 buscam testar o paradoxo controle e caos. Percebe-se na pergunta 1 que $66,67 \%$ dos entrevistados concordam que o prontuário pode aumentar o 
Revista de Administração Hospitalar, v.10, n.3, pp. 61-74, setembro/dezembro, 2013/ Marcelo Campara, Reinaldo Araujo Alkimin, Cristiana Fernandes De Muylder, Alexandre Teixeira Dias, José Marcos Carvalho de Mesquita, Jefferson La Falce

controle dos dados. Já na pergunta 9, identifica-se que mais de $44,44 \%$ dos respondentes acreditam que o PEP pode facilitar nas tarefas rotineiras. Na pergunta 16 a pesquisa constatou que $67,67 \%$ entendem que o PEP é necessário.

Percebe-se então que os testes do paradoxo 1 um em relação aos entrevistados foi confirmado.

Logo ocorre uma expectativa de controle na implantação do PEP, na amostra pesquisada.

Paradoxo dois: liberdade/escravidão

A pergunta 6 remete a dependência do usuário em relação ao sistema. Constatou-se que $33,33 \%$ acreditam que o sistema gerará mais liberdade do que escravidão.

Já na pergunta 10 que questiona se as atividades dos profissionais serão mais independentes, $38,89 \%$ concordam totalmente que o PEP gerará mais liberdade entre os profissionais na sua rotina de trabalho.

Com relação à burocratização dos processos na pergunta 17 , houve um empate. $33,33 \%$ dos entrevistados concordam parcialmente e totalmente que o PEP trará desburocratização, reafirmando o paradoxo de liberdade.

Paradoxo três: novo/obsoleto

Na pergunta 7 um total de 67,67\% dos entrevistados acreditam que o PEP já deveria ter sido implantado no Brasil, corroborando o paradoxo 'obsoleto'.

De acordo com a pesquisa, na pergunta 11, que questiona se o PEP pode gerar novos conhecimentos, $44,44 \%$ concordam que haverá geração de conhecimento, remetendo ao paradoxo "novo".

Neste caso percebe-se a junção dos dois paradoxos.

Paradoxo quatro: competência/incompetência

A questão 8 fala sobre o sentimento de incompetência caso o usuário não saiba operar o prontuário. $50 \%$ dos entrevistados concordam parcialmente.

A pergunta 18 questiona se os usuários precisarão de um programa de capacitação para operar o sistema e, $61,11 \%$ concordam totalmente com o questionamento, reforçando o paradoxo de competência.

Paradoxo cinco: eficiência/ineficiência

A pergunta 2 questiona se o PEP gerará menos esforço na execução das tarefas, e $61,11 \%$ dos entrevistados concordam com o paradoxo eficiência, ou seja o PEP ajudará na execução das tarefas.

Com relação à rapidez na execução das tarefas na questão $12,44,44 \%$ concordam parcialmente com a agilidade no processo. Ambas as perguntas reforçam o paradoxo eficiência.

Paradoxo seis: satisfação/criação de necessidades

A questão 13 aborda se o PEP agregará valor ao trabalho dos profissionais. 38,89\% acreditam totalmente que o sistema agregará valor ao seu trabalho, corroborando o paradoxo 'satisfação'. 
Revista de Administração Hospitalar, v.10, n.3, pp. 61-74, setembro/dezembro, 2013/ Marcelo Campara, Reinaldo Araujo Alkimin, Cristiana Fernandes De Muylder, Alexandre Teixeira Dias, José Marcos Carvalho de Mesquita, Jefferson La Falce

Em relação à saúde pessoal do paciente, pergunta 3, a maioria dos entrevistas $(27,785)$ acreditam que o programa é indiferente. Já a pergunta 19 questiona se o programa pode gerar futuras necessidades, e $50 \%$ dos entrevistados concordam totalmente.

Paradoxo sete: integração/isolamento

A questão 4 pergunta se o PEP pode gerar isolamento entre médico e paciente, e 33,33\% não concordam com a afirmativa. Ou seja, verificação do paradoxo integração.

Já na pergunta 14, que questiona sobre a integração entre os profissionais, 50\% concordam parcialmente que o PEP gerará mais integração. 
Revista de Administração Hospitalar, v.10, n.3, pp. 61-74, setembro/dezembro, 2013/ Marcelo Campara, Reinaldo Araujo Alkimin, Cristiana Fernandes De Muylder, Alexandre Teixeira Dias, José Marcos Carvalho de Mesquita, Jefferson La Falce

Paradoxo oito: engajamento/desengajamento

A pergunta 5 questiona se o sistema pode causar acomodação dos profissionais e gerou um empate. 33,33\% dos profissionais concordam e discordam parcialmente da afirmativa. Neste caso houve antagonismo entre os paradoxos engajamento e desengajamento.

Na pergunta 15 os entrevistados foram questionados se haverá ou não engajamento em prol do sistema. $67,67 \%$ concordam parcialmente que haverá engajamento dos profissionais em prol do sistema.

\section{Considerações Finais}

Por meio desta pesquisa, baseada no modelo de paradoxos proposto por Mick e Fournier (1998), pode-se verificar a expectativa de um grupo de profissionais de saúde em relação à implantação e utilização do Prontuário Eletrônico do Paciente - PEP.

A análise demonstrou uma predominância da parte positiva dos paradoxos: Controle, Liberdade, Competência, Eficiência, Satisfação, Integração e Engajamento. A presença dos paradoxos tidos como 'negativos' também foram encontrados, mesmo com menor intensidade, como uma preocupação dos usuários.

Os paradoxos negativos encontrados foram: obsoletismo, incompetência e criação de novas necessidades.

A presença dos paradoxos 'negativos' corrobora com a ideia de satisfação dos entrevistados. Todas as respostas tidas como 'negativas', de certa forma, se tornam positivas para a implantação do sistema.

A exemplo o paradoxo obsoleto, que reforça a ideia que o sistema já deveria ter sido implantado no país, ele é obsoleto com relação aos outros países que já adotam o programa a mais tempo.

Já o paradoxo incompetência, foi percebido na necessidade que os usuário terão de pesquisar e entender os procedimentos do novo programa. Com relação à criação de novas necessidades, a resposta se refere à mudança de rotina e geração de novas demandas para os usuários.

Não se trata de ineficiência do sistema, apenas a ideia de mudança de rotina. Desta forma, conclui-se que o fato dos paradoxos serem tidos como negativos não significam que eles são ruins ou que ocorra resistência à implantação do sistema.

A utilização dos paradoxos foi fundamental para classificar e agrupar as respostas e as expectativas dos usuários dentro de um cenário de inovação e mudanças.

Com a realização deste estudo percebeu-se avanços na reflexão da importância de novas pesquisas com o foco na avaliação do uso do PEP e ainda comparações de expectativas e benefícios percebidos após a implantação com foco nos paradoxos. Pode-se ainda sugerir como novo estudo a comparação de paradoxos apontados como negativos pelos usuários de dois cenários díspares como instituição privada com alta rentabilidade e instituição governamental baseada no Sistema Único de Saúde ou até mesmo em países diferentes. 
Revista de Administração Hospitalar, v.10, n.3, pp. 61-74, setembro/dezembro, 2013/ Marcelo Campara, Reinaldo Araujo Alkimin, Cristiana Fernandes De Muylder, Alexandre Teixeira Dias, José Marcos Carvalho de Mesquita, Jefferson La Falce

\section{Referências}

BRASIL. Governança no Sistema Único de Saúde (SUS) do Brasil: melhorando a qualidade do gasto público e gestão de recursos. Relatório No. 36601-BR. Disponível em: http://bvsms.saude.gov.br/bvs/IIIfis/pdf/Rogerio_Sugai.pdf. Acesso em 05/set/2013.

BUI, Alex A.T. et al. An XML Gateway to Patient Data for Medical Research Applications. Disponível em: <www.mii.ucla.edu/papers/bui_metmbs_2001b.pdf.

DE VRIES, M.K. . Organizational paradoxes: clinical approaches to management. New York: Norton. 1995

EISENHARDT, K. \&WESTCOTT, B. Paradoxical demands and the creation of excellence: the case of just-in-time manufacturing. In Quinn R. E. e Cameron K.S. (Eds.) Paradox and transformation: toward a theory of change in organization and management (pp. 81-121). Cambridge, MA: Ballinger. 1988

HATCH, M. \& EHRLICH, S. Spontaneous humour as an indicator of paradox and ambiguity in organizations. Organization Studies, 14(4), 539-560. 1993

MCDONALD, C.J., BARNETT, G.O. Medical Informatics: Computer Applications in Healt care. New York, 1990. p.181-218. 1990.

MASSAD, Eduardo; MARIN, Heimar de Fátima; AZEVEDO, Raymundo Soares. (2012). O prontuário eletrônico do paciente na assistência, informação e conhecimento médico. São Paulo: Edição do Autor, 2003. Disponível em <http://www.sbis.org.br/site/arquivos/prontuario.pdf>. Acesso em: 19 set. 2012.

MICK, D. \& FOURNIER, S. Paradoxes of Technology: consumer cognizance, emotions and coping strategies. Journal of Consumer Research, 25(20), 1998. 123-143.

MURNIGHAN, J. \& CONLON, D. The Dynamics of Intense Work Groups: A Study of British String Quarters. Administrative Science Quarterly, 36, 1991. 165-186.

PINTO, Virgínia Bentes. Prontuário eletrônico do paciente: documento técnico de informação e comunicação do domínio da saúde. R. Eletr. Bibliotecon. Ci. Inf., Florianópolis, n.21, $1^{\circ}$ sem. 2006.

SABELIS, I. Temporal paradoxes: Working with cultural diversity in organizations. In Koot, W., Sabelis, I., \& Ybema, S. (Eds). Contradictions in context: Puzzling over paradoxes in contemporary organizations (pp 171-192). Amsterdam: VU University Press. 1996.

SBIS. Sociedade Brasileira de Informática em Saúde. Disponível em: http://www.sbis.org.br/. Acesso em 19/09/2013.

SITTING, D.F. Grand challenges in medical informatics? Journal of the American Medical Informatics Association, Bethesda, v.1, n.5, p.412-413. 1994. 
Revista de Administração Hospitalar, v.10, n.3, pp. 61-74, setembro/dezembro, 2013/ Marcelo Campara, Reinaldo Araujo Alkimin, Cristiana Fernandes De Muylder, Alexandre Teixeira Dias, José Marcos Carvalho de Mesquita, Jefferson La Falce

SANTOS, Marcelo Rodrigues. Sistema de registro eletrônico de saúde baseado na norma ISO 13606: aplicações na Secretaria de Estado de Saúde de Minas Gerais. [Tese]. Universidade Federal de Minas Gerais, Belo Horizonte. 2011.

VAN BEMMEL, J. H.; MUSEN, M.A. Handbook of medical informatics. Netherlands: Springer-Verlag. 1997.

VINCE, R. \& BROUSSINE, M. Paradox, defense and attachment: accessing and working with emotions and relations underlying organizational change. Organizations Studies, 17(1), 1-21. 1996. 